\title{
Traditional Chinese medicine Ka-Sai-Ping suppresses the growths of gastric cancers via induction of autophagy
}

\author{
Mo-Li Zhu ${ }^{1, *}$, Jun-Xiu Lu ${ }^{2, *}$, Guo-Pin Pan ${ }^{1}$, Song Ping ${ }^{1}$, Fan-Rong Zhao ${ }^{3}$, Heng-Tian \\ $\mathbf{Q i}^{4}$, Hai-Ya Yu ${ }^{5}$, Xu Jian ${ }^{1}$, Guang-Rui Wan ${ }^{1}$ and Peng $\mathbf{L i}^{1}$ \\ ${ }^{1}$ College of Pharmacy, Xinxiang Medical University, Xinxiang, China \\ ${ }^{2}$ Department of Histology and Embryology, Xinxiang Medical University, Xinxiang, China \\ ${ }^{3}$ San-Quan College of Xinxiang Medical University, Xinxiang, China \\ ${ }^{4}$ Department of Cardiothoracic Surgery, The Third Affiliated Hospital, Xinxiang Medical University, Xinxiang, China \\ ${ }^{5}$ Department of Neurology, The People's Hospital of Xishui County, Huangang, Hubei, China \\ *These authors contributed equally to this work
}

Correspondence to: Peng Li, email: pengli@xxmu.edu.cn

Keywords: traditional Chinese medicine, Ka-Sai-Ping, gastric cancer, autophagy, apoptosis

Received: March 17, 2017 Accepted: May 10, $2017 \quad$ Published: May 20, 2017

Copyright: Zhu et al. This is an open-access article distributed under the terms of the Creative Commons Attribution License 3.0 (CC BY 3.0), which permits unrestricted use, distribution, and reproduction in any medium, provided the original author and source are credited.

\section{ABSTRACT}

Traditional Chinese medication is increasingly used to treat a wide range of human chronic diseases like cardiovascular diseases and cancers. This study was designed to explore whether ka-sai-ping (KSP), a novel traditional Chinese medicine developed by us, prevents gastric cancer growths and to investigate the underlying mechanism. The xenograft model of mouse gastric cancer was established by injecting MFCs into nude mouse subcutaneously. Cell autophagy was assessed by MDC staining. Lysosome and mitochondria were detected by Lyso-Tracker Red and Mito-Traker Green staining. Incubation of cultured mouse gastric cancer cell line MFCs with KSP for 48 hours, concentration-dependently reduced cell survivals and activated autophagy, which were accompanied with damaged lysosomes and mitochondria. In vivo studies indicated that KSP therapy $(20 \mathrm{ml} / \mathrm{kg} / \mathrm{day})$ for two weeks suppressed the growth of gastric cancer, increased the protein levels of LC3-II, beclin-1, cathepsin L, bcl-2, p53, and capase-3 in tumor tissues from the xenograft model of mouse gastric cancer. Importantly, all these effects induced by KSP were abolished by co-administration of autophagy inhibitor 3-MA. In conclusion, KSP activates cell autophagy to suppress gastric cancer growths. Clinically, KSP is potentially considered as a medicine to treat patients with gastric cancer.

\section{INTRODUCTION}

Gastric cancer or gastric carcinoma is a kind of malignant tumors worldwide [1]. Till now, chemotherapy is the mainstay approach for patients with gastric cancer [2]. However, gastric cancer usually develops resistance to chemotherapeutic drugs with a short life of disease control, and most patients would go to die within one or two years $[3,4]$. To combat the mortality rates from gastric adenocarcinoma, some new drugs are in want of.

Traditional Chinese medicine is widely used in China and other countries in East Asia [5, 6]. Typically, formulae are in consist of minerals or herbs, in which one represents the principal component, and others serve as adjuvants to increase the effects of principal component. In some formulae, multiple components could interact multiple targets and produce high efficacies.

The complexity of traditional Chinese medicine demonstrates that treatment protocols are able to be carefully designed to fight a disease. Therefore, we have generated a traditional Chinese compound prescription of xin-mai-jia to treat patients with atherosclerosis in China by improving endothelial function [7]. In this way, we developed a novel traditional Chinese compound 
prescription of ka-sai-ping (KSP) to prevent gastric cancer in light of the theory of traditional Chinese medicine and the pathophysiological mechanism of gastric cancer.

\section{RESULTS}

\section{KSP reduces gastric cancer cell survivals}

To investigate the effects of KSP on gastric cancer cell growth, mouse gastric cancer cell line (MFC) was cultured and treated with $\operatorname{KSP}(2,6.7,20 \mathrm{mg} / \mathrm{l})$ for 48 hours. Cyclophosphamide (CTX, $100 \mathrm{mg} / \mathrm{l}$ ) and Chinese medicine injection Aidi $(15 \mathrm{ml} / \mathrm{l})$, served as positive controls. Cell viabilities were detected by CCK8 assay. As shown in Figure 1A, KSP, similar to CTX and Aidi, concentration-dependently reduced cell viabilities of MFCs. These data indicate that KSP may inhibit gastric cancer growth.

\section{KSP increases cell autophagy in cultured MFCs}

Autophagy is involved in the biological process of gastric cancer [8]. Thus, we determined the effects of KSP on autophagy in MFCs by MDC staining and IFC analysis. As shown in Figure 1B-1D, KSP, as well as CTX and Aidi, increased the formation of autophagosome and the levels of LC3-II protein in MFCs, suggesting that KSP activates autophagy in gastric cancer cells.

\section{KSP induces the formations of mitophagosome and mitolysosome in MFCs}

Both mitochondria and lysosome are critical to the formation of autophagosome in cells [9, 10]. We next detected mitophagosome and mitolysosome by MitoTracker Green and Lyso-Tracker Red. As indicated in Figure 2A-2C, KSP, CTX, and Aidi increased the fluorescence intensities of Mito-Tracker Green and Lyso-Tracker Red in MFCs, further supporting that KSP increases cell autophagy in gastric cancer cells.

\section{KSP suppresses the in situ growth of gastric cancer in mice}

To evaluate the effects of KSP on tumor growth in vivo, we generated the xenograft model of mouse gastric cancer by injecting MFCs into nude mouse subcutaneously. As shown in Figure 3A, transplantation of MFCs into nude mice induced the in situ formation of gastric cancer. Two-week treatments of KSP dramatically reduced the tumor weight (Figure 3B). HE staining

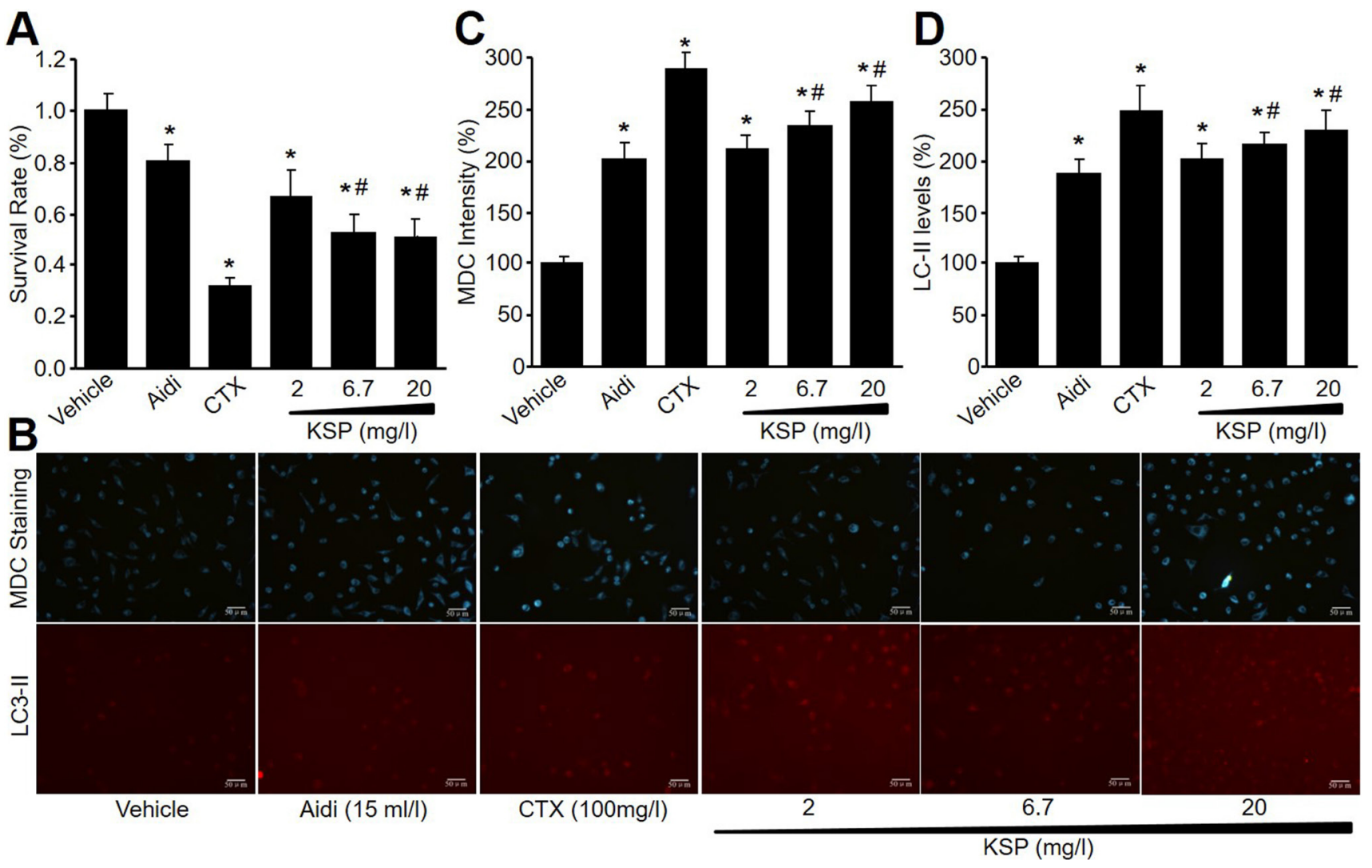

Figure 1: KSP reduces cell survival and increases autophagy in cultured MFCs. MFCs were incubated with Aidi (15 ml/1), CTX (100 mg/l), and KSP (2, 6.7, $20 \mathrm{mg} / \mathrm{l})$ for 48 hours. Cell survival was detected by CCK 8 assay in (A). Autophagy was determined by MDC staining and CY3 staining in (B). The quantitative analyses of autophagy in (C) and LC3-II in (D) were performed in pictures from B. $\mathrm{N}$ is 3 in each group. ${ }^{*} P<0.05 \mathrm{VS}$ vehicle, ${ }^{\#} P<0.05 \mathrm{VS} 2 \mathrm{mg} / \mathrm{ml} \mathrm{KSP}$. 
indicated that KSP reduced the cell numbers of MFCs in tumor tissues (Figure 3C). These data demonstrate that $\mathrm{KSP}$ is considered as an anti-cancer drug on gastric cancer.

\section{KSP via induction of autophagy inhibits gastric cancer growth in vivo}

To determine the role of autophagy in KSP-reduced gastric cancer growth in mice, 3-MA was administrated into nude mice to block autophagy [11]. As depicted in Figure 3B-3C, co-treatment of 3-MA abolished the inhibitory effects of KSP on gastric cancer growth including tumor weight and cell numbers. The role of autophagy in KSP-inhibited gastric cancer growth was further confirmed by assaying the protein levels of LC3-II and beclin-1 in tumor tissue. As shown in Figure 4A-4B, KSP significantly increased the levels of LC3-II and beclin-1 in tumor tissues, which was ablated by 3-MA, suggesting that KSP via induction of autophagy inhibits gastric cancer growth in vivo.

\section{KSP increases cathepsin $\mathrm{L}$ in gastric cancer in vivo}

Cathepsin L plays a key in the switch from autophagy to apoptosis $[12,13]$. Thus, we measured the levels of cathepsin L in tumor tissue by IHC analysis (Figure 5A) and Western blot analysis (Figure 5B). The expressional level of cathepsin L was enhanced by KSP. However, co-administration of 3-MA abrogated the effects of KSP on the upregulation of cathepsin L protein in tumor tissue.

\section{KSP induces apoptosis of gastric cancer in mice}

Emerging evidence suggests that cross-talk occurs between autophagic and apoptotic pathways [14]. Thus, we also examined the role of apoptosis in KSP-inhibited gastric cancer growth. The apoptosis-related proteins including p53, bcl-2, and caspase- 3 were assayed by analyses of IHC and western blot. As indicated in Figure 6A-6C, KSP increased the apoptotic protein levels

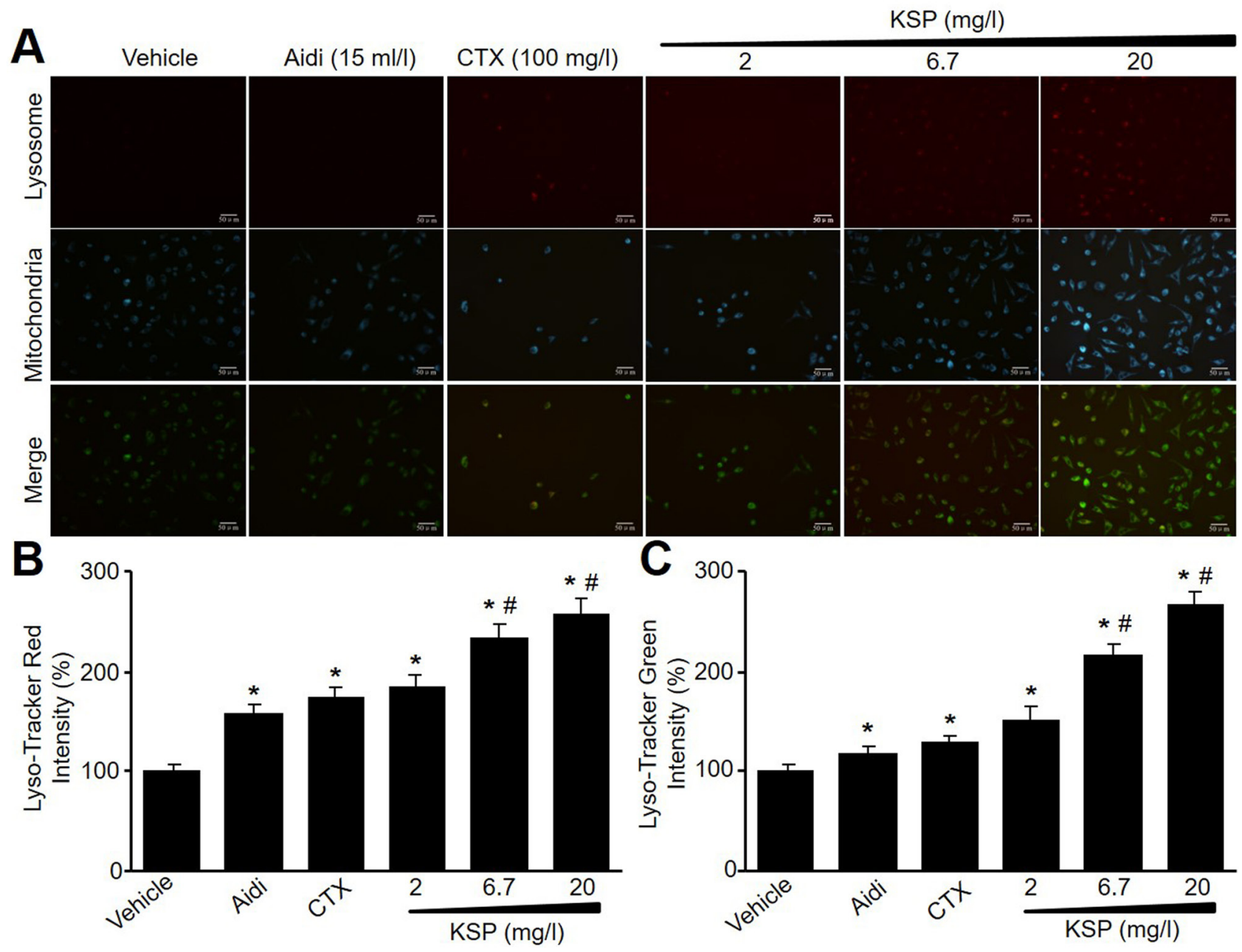

Figure 2: KSP induces the formations of mitophagosome and mitolysosome in MFCs. MFCs were incubated with Aidi $(15 \mathrm{ml} / \mathrm{l})$, CTX (100 mg/l), and KSP (2, 6.7, $20 \mathrm{mg} / \mathrm{l})$ for 48 hours. (A) Mitochondria and lysosome were detected by Lyso-Tracker Red and Mito-Tracker Green. The presented picture is a representative picture from three independent experiments. (B and $\mathbf{C})$ The quantitative analyses of lysosomal in B and mitochondria in $\mathrm{C}$ were performed. $\mathrm{N}$ is 3 in each group. ${ }^{*} P<0.05 \mathrm{VS}$ vehicle, ${ }^{*} P<0.05 \mathrm{VS} 2 \mathrm{mg} / \mathrm{ml} \mathrm{KSP}$. 

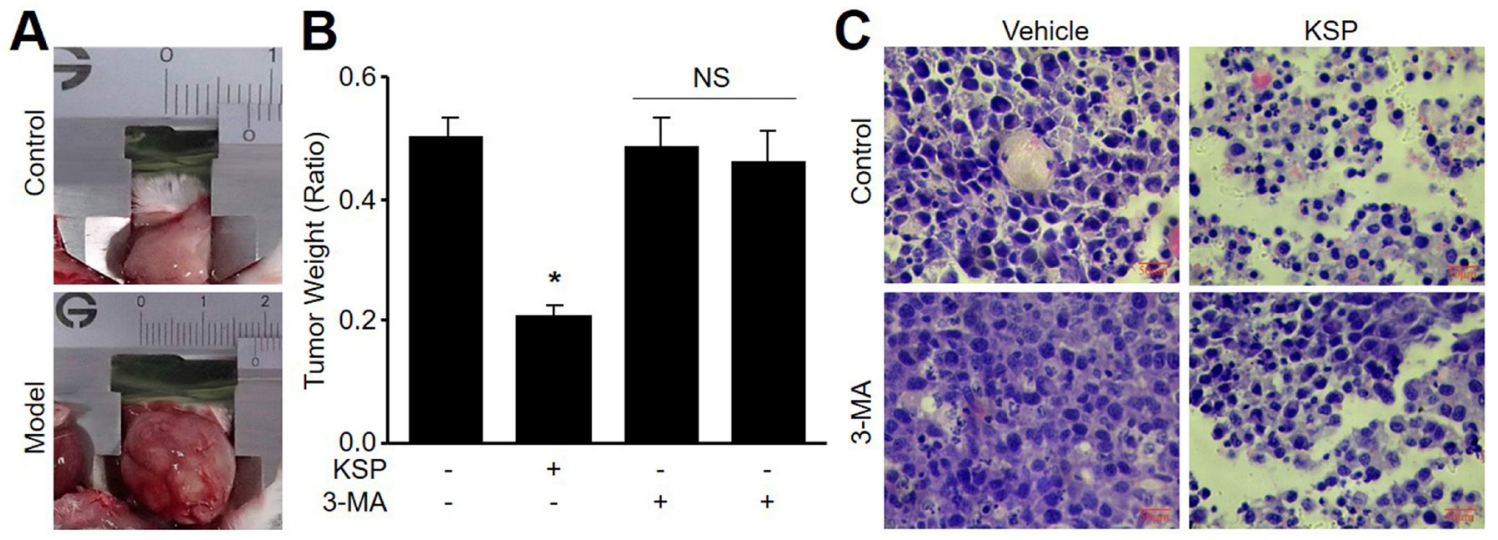

Figure 3: KSP suppresses the growth of gastric cancer in mice. Nude mice received MFCs transplantations and then were treated with KSP (20 ml/kg/day) in presence or absence of 3-MA $(10 \mathrm{mg} / \mathrm{kg} /$ day) for two weeks. (A) Morphology of gastric cancer in mice. (B) Tumor weight of gastric cancer in mice. (C) HE staining of gastric cancer in mice. 5-10 mice in each group. ${ }^{*} P<0.05$ VS Control. NS indicates no significance.

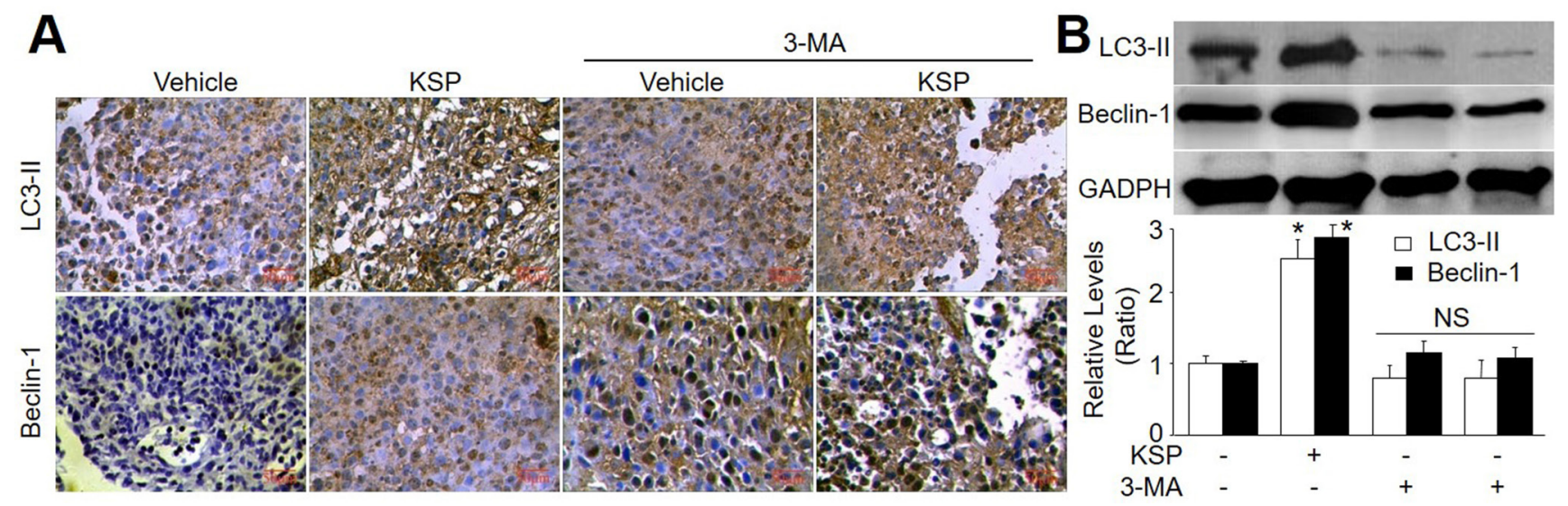

Figure 4: KSP increases autophagy of gastric cancer in mice. Nude mice received MFCs transplantations and then were treated with KSP (20 ml/kg/day) in presence or absence of 3-MA (10 mg/kg/day) for two weeks. (A) IHC analyses of LC3-II and beclin-1 were performed in tissues of gastric cancer from mice. (B) The levels of LC3-II and beclin-1 were determined in tumor tissues of gastric cancer in mice by Western blot. 5-10 mice in each group. ${ }^{*} P<0.05$ VS Control. NS indicates no significance.
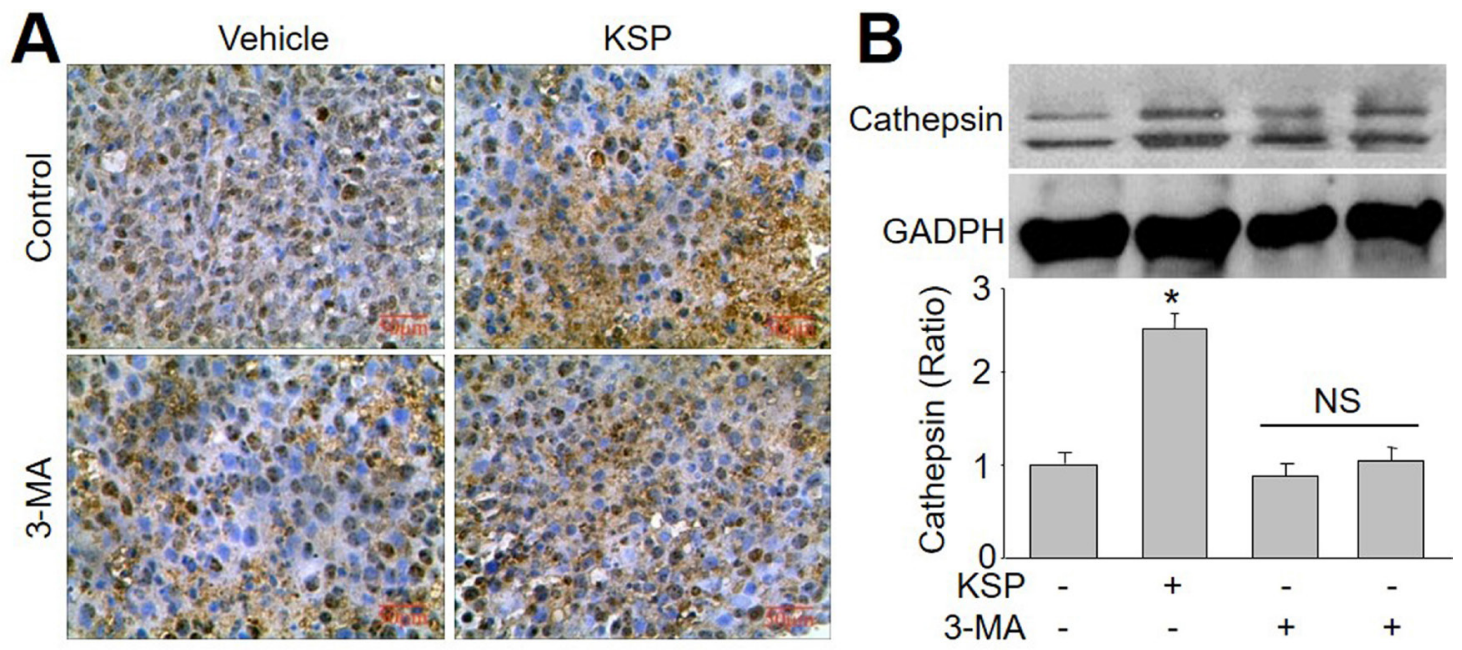

Figure 5: KSP increases the switch from autophagy to apoptotic death of gastric cancer in mice. Nude mice received MFCs transplantations and then were treated with KSP (20 ml/kg/day) in presence or absence of 3-MA (10 mg/kg/day) for two weeks. (A) IHC analysis of cathepsin L was performed in tumor of gastric cancer in mice. (B) The levels of cathepsin L was determined in tumor tissues of gastric cancer in mice by Western blot. 5-10 mice in each group. ${ }^{*} P<0.05$ VS Control. NS indicates no significance. 
of p53 and caspase-3, but decreased the level of bcl-2, which functions as anti-apoptosis [15]. These effects of KSP on apoptosis-related proteins were attenuated by 3-MA, indicating that KSP-induced apoptosis of gastric cancer is dependent on autophagy.

\section{DISCUSSION}

Gastric cancer is the third leading cause of cancer death in the world $[16,17]$. Although the survival rate of gastric cancer patients is improved with the development of chemotherapy in the past years, the long-term survival rates of these patients are still not satisfied. In this study, we developed a new prescription named after KSP and observed that KSP inhibits tumor growth in the xenograft model of mouse gastric cancer.

Mechanically, the anti-cancer effects of KSP is mediated by induction of autophagy in gastric cancer cells. In the process of autophagy, a phagophore from endoplasmic reticulum or plasma membrane forms autophagosomes, which depend on beclin-2, LC3-II, etc [18]. In this study, we observed that KSP promoted

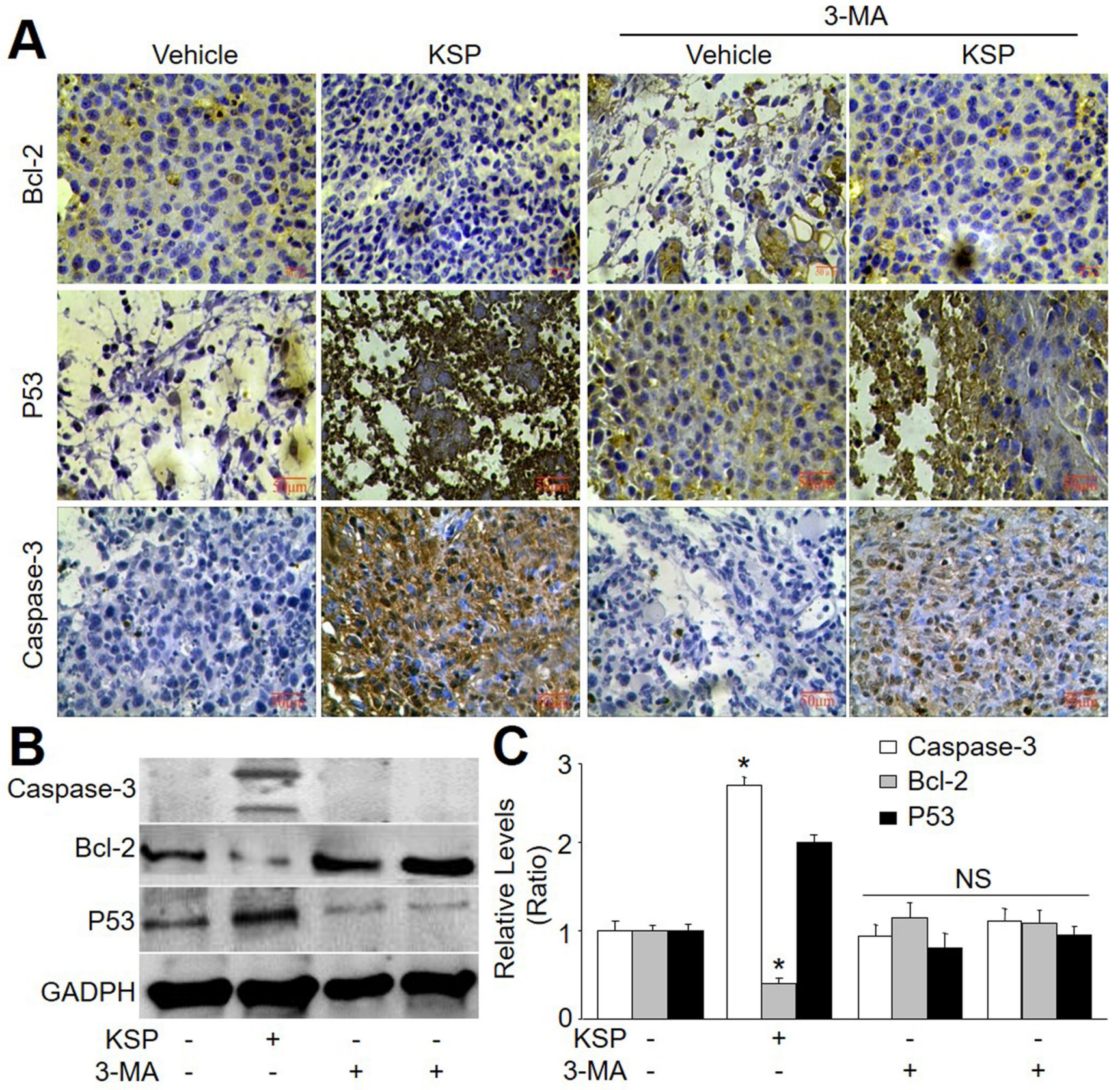

Figure 6: KSP induces cell apoptosis of gastric cancer in mice. Nude mice were received MFCs transplantations and then were treated with KSP (20 ml/kg/day) in presence or absence of 3-MA (10 mg/kg/day) for two weeks. (A) IHC analyses of bcl-2, p53, and caspase-3 were performed in tumor of gastric cancer in mice. (B) The levels of bcl-2, p53, and caspase-3 were determined in tumor tissues of gastric cancer in mice by Western blot. (C) Quantitative analysis of results from B. 5-10 mice in each group. ${ }^{*} P<0.05$ VS Control. NS indicates no significance. 
the formation of autophagosomes, mitophagosome and mitolysosome, and increased beclin-2 and LC3-II protein levels. This raised subsequent reduction of cell survival by destroying the cytosol and organelles, leading to cell death. KSP functions as an autophagy inducer in gastric cancer cells. Actually, some papers have reported that traditional Chinese medicine is able to regulate autophagy $[19,20]$, supporting our conclusion.

In summary, we have developed a novel traditional Chinese medicine of KSP to prevent the growth of gastric cancer in mice. The underlying mechanism is related to the induction of autophagy of gastric cancer cells. Our results provided another choice to prevent and treat patients with gastric cancer or other gastrointestinal tumors clinically.

\section{MATERIALS AND METHODS}

\section{Animals and Materials}

KSP is a Chinese medicinal formulation that is available in capsule form. The formula contains lucid ganoderma, silymarin, resveratrol, hedyotis diffusa, epimedium, oysters, and codonopsis pilosula. KSP crude drugs were purchased from Tong-Ren-Tang Company (Beijing, China). Aidi injection was purchased from YiBai Pharmaceutical Company (Guizhou, China). CCK8 kit was obtained from Dojindo Laboratories (Kumamoto, Japan). Lyso-Tracker Red C1046 and Mito-Tracker Green C1048 were brought from Beyotime Biotechnology (Shanghai, China). Primary antibodies against bcl-2 (ab59348), p53 (ab31333), capase-3 (ab2302), beclin-1 (ab62557), LC3-II (ab48394), and cathepsin L (ab6314) were purchased from Abcam (United States). All female BAL b/c nude mice were purchased from Institute of Laboratory Animal Sciences, Cams\&Pumc; Beijing, China.

\section{Cell culture and analysis for cell viability}

The mouse gastric cancer cell line (MFC) was purchased from the Cell Bank of the Chinese Academy of Medical Sciences, Beijing, China. Cell viability was measured by CCK kit according to the manufacture's instruction as described previously [21].

\section{MDC staining of autophagic vacuoles}

MDC staining of autophagic vacuoles was performed for autophagy analysis as previously described [22].

\section{Establishment of the xenograft model of gastric cancer in mice}

The xenograft model of gastric cancer was established as described previously [23].). Following two days of growth, animals were randomly divided into 4 groups. Group 1: Vehicle group; Group 2: KSP group; Group 3: 3-MA group; Group 4: KSP plus 3-MA group. Mice in group 1 intragastrically administrated with saline for 2 weeks. Mice in group 2 received gavage with KSP $(20 \mathrm{ml} / \mathrm{kg} /$ day $)$ for 2 weeks. Mice in group 3 received treatment of 3-MA $(10 \mathrm{mg} / \mathrm{kg} /$ day $)$ in drinking water for 2 weeks. Mice in group 4 received KSP plus 3-MA for 2 weeks. This study was carried out in strictly accordance with the recommendations in the Guide for the Care and Use of Laboratory Animals of the National Institutes of Health. The protocol was approved by the Committee on the Ethics of Animal Experiments of Xinxiang Medical University.

\section{Immunohistochemistry (IHC)}

As described previously [24], the tissue was fixed in $4 \%$ paraformaldehyde overnight, and then processed, embedded in paraffin, and sectioned at $4 \mu \mathrm{m}$. Semiquantitative analysis of tissue immunoreactivity was done as described previously with modifications [25].

\section{Western blot analysis}

As described previously [26, 27], total proteins were subjected to Western blots using ECL-Plus.

\section{Statistical analysis}

Values are expressed as mean \pm SEM. Statistical comparisons were performed by using one-way ANOVA. Intergroup differences were analyzed using Bonferroni's post-test. Analysis of dose-course studies was performed with repeated measures ANOVA. $P$ values less than 0.05 were considered as significant.

\section{Authors' contributions}

Mo-Li Zhu and Jun-Xiu Lu performed all experiments. Guo-Pin Pan, Song Ping, Fan-Rong Zhao, Heng-Tian Qi, and Hai-Ya Yu partially performed some experiments. $\mathrm{Xu}$ Jian and Guang-Rui Wan gave some critical suggestions to this project. Peng Li convinced the project and wrote the manuscript.

\section{CONFLICTS OF INTEREST}

None.

\section{FUNDING}

This project was supported by National Natural Science Foundation of China (81570723, 81673423), Natural Science Foundation of Henan Province 
(162300410216), the Science and Technology Research Project of Henan Province (121100910300 and 132102310247), and the Research Fund of Xinxiang Medical University (2014QN153 and ZD2011-30).

\section{REFERENCES}

1. Xu J, Liu D, Niu H, Zhu G, Xu Y, Ye D, Li J, Zhang Q. Resveratrol reverses Doxorubicin resistance by inhibiting epithelial-mesenchymal transition (EMT) through modulating PTEN/Akt signaling pathway in gastric cancer. J Exp Clin Cancer Res. 2017; 36:19.

2. Kanat O, O’Neil BH. Metastatic gastric cancer treatment: a little slow but worthy progress. Med Oncol. 2013; 30:464.

3. Wagner AD, Grothe W, Haerting J, Kleber G, Grothey A, Fleig WE. Chemotherapy in advanced gastric cancer: a systematic review and meta-analysis based on aggregate data. J Clin Oncol. 2006; 24:2903-09.

4. Oba K, Paoletti X, Bang YJ, Bleiberg H, Burzykowski T, Fuse N, Michiels S, Morita S, Ohashi Y, Pignon JP, Rougier P, Sakamoto J, Sargent D, et al, and GASTRIC (Global Advanced/Adjuvant Stomach Tumor Research International Collaboration) Group. Role of chemotherapy for advanced/ recurrent gastric cancer: an individual-patient-data metaanalysis. Eur J Cancer. 2013; 49:1565-77.

5. Chen KJ, Lu AP. Situation of integrative medicine in China: results from a national survey in 2004. Chin J Integr Med. 2006; 12:161-65.

6. Wang L, Zhou GB, Liu P, Song JH, Liang Y, Yan XJ, Xu F, Wang BS, Mao JH, Shen ZX, Chen SJ, Chen Z. Dissection of mechanisms of Chinese medicinal formula RealgarIndigo naturalis as an effective treatment for promyelocytic leukemia. Proc Natl Acad Sci USA. 2008; 105:4826-31.

7. Yin YL, Zhu ML, Wan J, Zhang C, Pan GP, Lu JX, Ping S, Chen Y, Zhao FR, Yu HY, Guo T, Jian X, Liu LY, et al. Traditional Chinese medicine xin-mai-jia recouples endothelial nitric oxide synthase to prevent atherosclerosis in vivo. Sci Rep. 2017; 7:43508.

8. Zhou H, Yuan M, Yu Q, Zhou X, Min W, Gao D. Autophagy regulation and its role in gastric cancer and colorectal cancer. Cancer Biomark. 2016; 17:1-10.

9. Nguyen TN, Padman BS, Usher J, Oorschot V, Ramm G, Lazarou M. Atg8 family LC3/GABARAP proteins are crucial for autophagosome-lysosome fusion but not autophagosome formation during PINK1/Parkin mitophagy and starvation. J Cell Biol. 2016; 215:857-74.

10. Gomez-Suaga P, Paillusson S, Stoica R, Noble W, Hanger DP, Miller CC. The ER-Mitochondria Tethering Complex VAPBPTPIP51 Regulates Autophagy. Curr Biol. 2017; 27:371-85.

11. Liu H, He Z, Simon HU. Targeting autophagy as a potential therapeutic approach for melanoma therapy. Semin Cancer Biol. 2013; 23:352-60.

12. Li L, Gao L, Song Y, Qin ZH, Liang Z. Activated cathepsin $\mathrm{L}$ is associated with the switch from autophagy to apoptotic death of SH-SY5Y cells exposed to 6-hydroxydopamine. Biochem Biophys Res Commun. 2016; 470:579-85.

13. Liu WJ, Shen TT, Chen RH, Wu HL, Wang YJ, Deng JK, Chen QH, Pan Q, Huang Fu CM, Tao JL, Liang D, Liu HF. Autophagy-Lysosome Pathway in Renal Tubular Epithelial Cells Is Disrupted by Advanced Glycation End Products in Diabetic Nephropathy. J Biol Chem. 2015; 290:20499-510.

14. Maiuri MC, Le Toumelin G, Criollo A, Rain JC, Gautier F, Juin P, Tasdemir E, Pierron G, Troulinaki K, Tavernarakis N, Hickman JA, Geneste O, Kroemer G. Functional and physical interaction between $\mathrm{Bcl}-\mathrm{X}(\mathrm{L})$ and a $\mathrm{BH} 3$-like domain in Beclin-1. EMBO J. 2007; 26:2527-39.

15. Hatok J, Racay P. Bcl-2 family proteins: master regulators of cell survival. Biomol Concepts. 2016; 7:259-70.

16. Ajani JA, Bentrem DJ, Besh S, D’Amico TA, Das P, Denlinger C, Fakih MG, Fuchs CS, Gerdes H, Glasgow RE, Hayman JA, Hofstetter WL, Ilson DH, et al, and National Comprehensive Cancer Network. Gastric cancer, version 2.2013: featured updates to the NCCN Guidelines. J Natl Compr Canc Netw. 2013; 11:531-46.

17. Lee JH, Kim JG, Jung HK, Kim JH, Jeong WK, Jeon TJ, Kim JM, Kim YI, Ryu KW, Kong SH, Kim HI, Jung HY, Kim YS, et al. Clinical practice guidelines for gastric cancer in Korea: an evidence-based approach. J Gastric Cancer. 2014; 14:87-104.

18. Kroemer G, Levine B. Autophagic cell death: the story of a misnomer. Nat Rev Mol Cell Biol. 2008; 9:1004-10.

19. Wang SF, Wu MY, Cai CZ, Li M, Lu JH. Autophagy modulators from traditional Chinese medicine: mechanisms and therapeutic potentials for cancer and neurodegenerative diseases. J Ethnopharmacol. 2016; 194:861-76.

20. Law BY, Mok SW, Wu AG, Lam CW, Yu MX, Wong VK. New Potential Pharmacological Functions of Chinese Herbal Medicines via Regulation of Autophagy. Molecules. 2016;21:359.

21. Zhang M, Pan Y, Dorfman RG, Chen Z, Liu F, Zhou Q, Huang S, Zhang J, Yang D, Liu J. AR-42 induces apoptosis in human hepatocellular carcinoma cells via HDAC5 inhibition. Oncotarget. 2016; 7:22285-94. https://doi. org/10.18632/oncotarget.8077.

22. Wang R, Zhang Q, Peng X, Zhou C, Zhong Y, Chen X, Qiu Y, Jin M, Gong M, Kong D. Stellettin B Induces G1 Arrest, Apoptosis and Autophagy in Human Non-small Cell Lung Cancer A549 Cells via Blocking PI3K/Akt/mTOR Pathway. Sci Rep. 2016; 6:27071.

23. Lin Y, Zhai E, Liao B, Xu L, Zhang X, Peng S, He Y, Cai $\mathrm{S}$, Zeng Z, Chen M. Autocrine VEGF signaling promotes cell proliferation through a PLC-dependent pathway and modulates Apatinib treatment efficacy in gastric cancer. Oncotarget. 2017; 8:11990-2002. https://doi.org/10.18632/ oncotarget.14467.

24. Yang JJ, Li P, Wang F, Liang WJ, Ma H, Chen Y, Ma ZM, Li QZ, Peng QS, Zhang Y, Wang SX. Activation of activator protein 2 alpha by aspirin alleviates atherosclerotic plaque 
growth and instability in vivo. Oncotarget. 2016; 7:52729 39. https://doi.org/10.18632/oncotarget.10400.

25. Wang S, Zhang M, Liang B, Xu J, Xie Z, Liu C, Viollet B, Yan D, Zou MH. AMPKalpha2 deletion causes aberrant expression and activation of $\mathrm{NAD}(\mathrm{P}) \mathrm{H}$ oxidase and consequent endothelial dysfunction in vivo: role of $26 \mathrm{~S}$ proteasomes. Circ Res. 2010; 106:1117-28.

26. Li P, Yin YL, Guo T, Sun XY, Ma H, Zhu ML, Zhao FR, $\mathrm{Xu}$ P, Chen Y, Wan GR, Jiang F, Peng QS, Liu C, et al. Inhibition of Aberrant MicroRNA-133a Expression in Endothelial Cells by Statin Prevents Endothelial Dysfunction by Targeting GTP Cyclohydrolase 1 in Vivo. Circulation. 2016; 134:1752-65.

27. Wang S, Zhang C, Zhang M, Liang B, Zhu H, Lee J, Viollet B, Xia L, Zhang Y, Zou MH. Activation of AMPactivated protein kinase $\alpha 2$ by nicotine instigates formation of abdominal aortic aneurysms in mice in vivo. Nat Med. 2012; 18:902-10. 